International Journal of Dentistry and Oral Science (JDOS)

ISSN: 2377-8075

\title{
ISW for the Treatment of Adult Angle Class I Crowding with Acceptable Lateral Profile Case
}

Tian-yu OU-YANG ${ }^{1}$, Jian-hong YU ${ }^{2,3^{*}}$, Ya-yu TSAI ${ }^{4}$, Chien-chih $\mathrm{YU}^{5}$

${ }^{1}$ Resident, Department of Orthodontics, China Medical University Hospital, Taichung, 40402, Taiwan, R.O.C.

${ }^{2}$ Associate Professor, School of Dentistry, College of Medicine, China Medical University, Taichung, 40402, Taiwan, R.O.C.

${ }^{3}$ Dean, Department of Orthodontics, China Medical University Hospital, Taichung, 40402, Taiwan, R.O.C.

${ }^{4}$ Attending, Department of Orthodontics, China Medical University Hospital, Taichung, 40402, Taiwan, R.O.C.

${ }^{5}$ Associate Professor, School of Pharmacy, College of Pharmacy, China Medical University, Taichung, 40402, Taiwan, R.O.C

\begin{abstract}
This article describes a 34-year-old adult female with chief complaint of poor dental alignment. She presented with Angle Class I crowding with acceptable lateral profile and had been treated with a non-extraction approach. The diagnosed after examination and analysis was Angle Class I malocclusion on Class I skeletal base with deep overbite and severe crowding. Treatment plan was carried out to preserve premolars and create space by using the MEAW technique, combined with stripping for gaining more space and IME for anchorage control and bite raising. In addition to the correction of deep bite, a better occlusion and a favorable facial profile were achieved.
\end{abstract}

Keywords: Angle Class I malocclusion; Severe Crowding; Non-Extraction; MEAW.

\section{*Corresponding Author:}

Dr. Jian-Hong YU,

Associate Professor, School of Dentistry, College of Medicine, and

Dean, Department of Orthodontics, China Medical University Hospi-

tal, Taichung, 40402, Taiwan, R.O.C.

E-mail: kenkoyu@mail.cmu.edu.tw

Received: June 23, 2015

Accepted: July 29, 2015

Published: August 03, 2015

Citation: Tian-yu OU-YANG, Jian-hong YU, Ya-yu TSAI, Chien-chih YU (2015) ISW for the Treatment of Adult Angle Class I Crowding with Acceptable Lateral Profile Case. Int J Dentistry Oral Sci. 2(8), 115-119. doi: http://dx.doi.org/10.19070/2377-8075-1500025

Copyright: Jian-hong YU ${ }^{\odot} 2015$. This is an open-access article distributed under the terms of the Creative Commons Attribution License, which permits unrestricted use, distribution and reproduction in any medium, provided the original author and source are credited.

\section{Introduction}

The main strategy to treat Angel Class I malocclusion combined with severe crowding is to create space for tooth alignment [1]. Although tooth extraction can result in predicted outcomes, this method requires the sacrifice of healthy teeth. For patients who refuse tooth extraction, an alternative approach must be undertaken. Other commonly used processes include flaring out of anterior teeth, transverse arch expansion, molar distalization, interproximal enamel reduction, and the use of multiple-loop edgewise archwire (MEAW) technique [2]. To sustain the original lateral profile, flaring out of anterior teeth was not used in this case seeking for treatment. Given the circumstance that patient refused tooth extraction, the treatment was completed with the use of ISW and MEAW technique to achieve a favorable outcome, but without flaring out of anterior teeth.

\section{Objective}

To describe the experience of using ISW (Improved Super-elastic Ti-Ni alloy wire, developed by Tokyo Medical and Dental University) to treat a case with Angle Class I and achieved an acceptable lateral profile dental crowding.

\section{Case Presentation}

An adult female aged 34 years who came to our clinic with a chief complaint of poor dental alignment (Figure 1, Figure 2, Figure 3). Clinical examination revealed that she had a deep overbite and dental crowding. ISW was used to level and relieve the crowding, followed by intermaxillary elastics (IME) to raise the bite. The use of MEAW technique enabled to gain space enough for adjusting the inclination of anterior teeth. The total active treatment period was 2 years and 4 months. The panoramic radiographs before active treatment showed that \#18, \#28, \#38, and \#48 were impacted teeth and \#25 had previous root canal treatment (Figure 4). In Cephalometric analysis, the measured angles of SNA, SNB, ANB, U1-FH were $79.0^{\circ}, 75.1^{\circ}, 3.9^{\circ}$, and $107.7^{\circ}$, respectively. The measured mandibular plane (L1 to $\mathrm{Md}$ ) and $\mathrm{Md}$ plane angles were $102.8^{\circ}$ and $25.5^{\circ}$ (Figure 5, Table 1). Arch length discrepancies in the UR (upper-right) quarter were $3.0 \mathrm{~mm}$ and $5.0 \mathrm{~mm}$ in UL (upper-left) and LL (lower-left), and also the LR (lower-right) arch. The diagnosis was Angle Class I malocclusion on a Class I skeletal base. The treatment planning involved a complete fixed orthodontic appliance without extracting the teeth \#18, \#38, and \# 48. 
Table 1.

\begin{tabular}{|c|c|c|c|c|}
\hline & Value & Mean. & S.D. & Diff \\
\hline Facial angle & 84.6 & 84.83 & 3.05 & -0.20 \\
\hline Convexity & 7.6 & 7.58 & 4.95 & 0.06 \\
\hline A-B plane & -6.8 & -4.81 & 3.50 & -2.02 \\
\hline Mandibular plane & 25.5 & 28.81 & 5.23 & -3.35 \\
\hline Y-axis & 63.0 & 65.38 & 5.63 & -2.37 \\
\hline Occlusal plane & 11.5 & 11.42 & 3.64 & 0.06 \\
\hline Interincisal & 124.0 & 124.09 & 7.63 & -0.12 \\
\hline L-I to Occlusal & 26.8 & 23.84 & 5.28 & 2.97 \\
\hline L-1 to Mandibular & 102.8 & 96.33 & 5.78 & 6.50 \\
\hline U-1 to A-P plane & 9.2 & 8.92 & 1.88 & 0.29 \\
\hline FMIA & 51.7 & 54.63 & 6.47 & -2.92 \\
\hline FH to SN plane & 9.2 & 6.19 & 2.89 & 3.00 \\
\hline SNA & 79.0 & 82.32 & 3.45 & -3.33 \\
\hline SNB & 75.1 & 78.90 & 3.45 & -3.80 \\
\hline SNA-SNB diff. & 3.9 & 3.39 & 1.77 & 0.50 \\
\hline U-1 to N-P plane & 11.3 & 11.74 & 2.73 & -0.42 \\
\hline U-1 to FH plane & 107.7 & 111.13 & 5.54 & -3.39 \\
\hline U-1 to SN plane & 98.6 & 104.54 & 5.55 & -598 \\
\hline Gonial angle & 120.1 & 122.23 & 4.61 & -2.10 \\
\hline Ramus inclination & 85.3 & 87.07 & 4.40 & -1.74 \\
\hline
\end{tabular}

Figure 1.

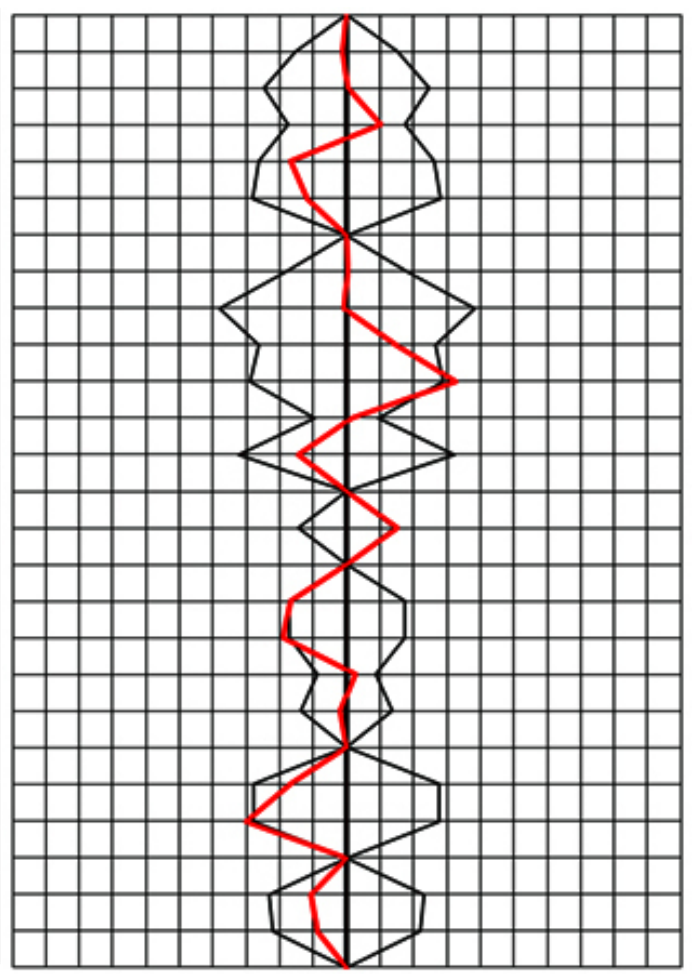

Figure 2.

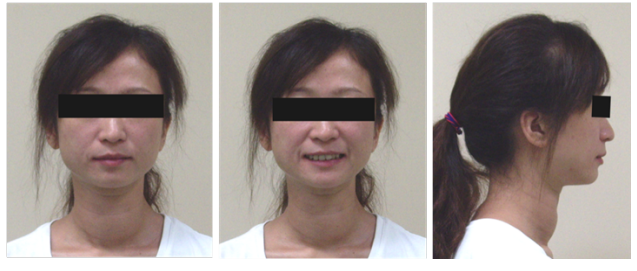

Figure 3.

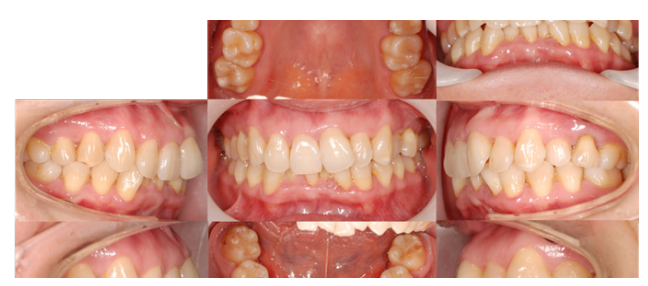

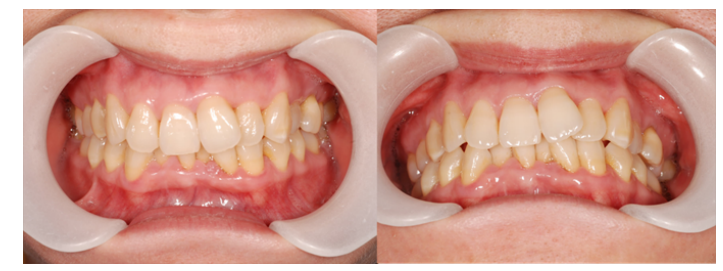

Figure 4 .

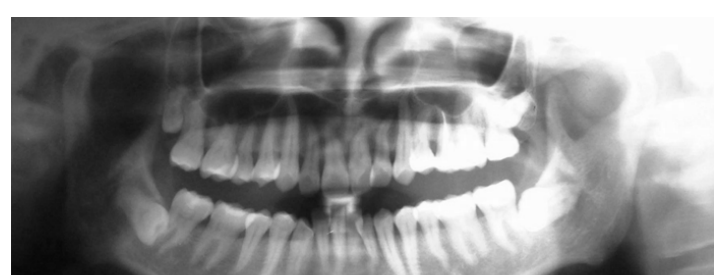

Figure 5 .
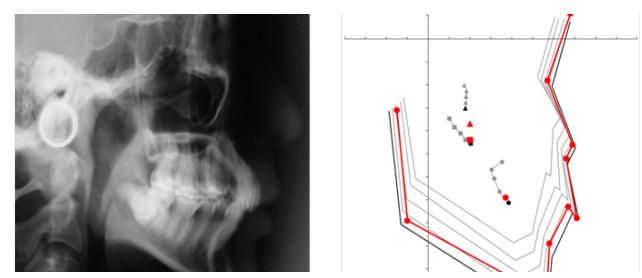

\section{Treatment Progress}

After the $3^{\text {rd }}$ molar extractions over UR, LL and LR quarters, 0.018 slot brackets (Roth-system) were placed on all teeth. The active treatment was started with $0.016 \times 0.022 \mathrm{ISW}$ wire to level the upper and lower arches, followed by bilateral Class II IME to raise the bite. Open coil spring was also placed to create space between teeth \#32 and \#41 (Figure 6, Figure 7). At the $7^{\text {th }}$ month of treatment, MEAW technique was applied over UR, UL, and LL arches. The strategy of IME allowed the anchorage of bilateral short Class II to be enforced by MEAW (from canine to lower first premolar) (Figure 8).

At the $24^{\text {th }}$ months, anterior teeth were well aligned; however, mild uneven marginal ridge relation with posterior teeth remained after MEAW technique. Thus, a new plain ISW wire $(0.016 \times 0.022)$ was used to replace the MEAW archwire for leveling. The use of IME was changed from for teeth \#14 and \#44, as well as teeth \#23, \#24, \#33, and \#44 for improving interdigitation (Figure 9). After 2 months of post-MEAW leveling, alignate impression was made for fabrication of orthodontic retainers and the placement of IME was changed to teeth \#13, \#43, and \#44 as well as \#23, 
Figure 6.

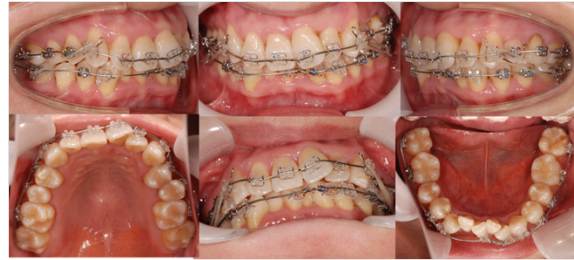

Figure 9 (24 months).

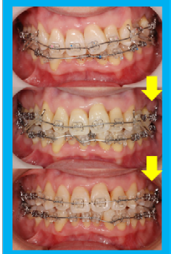

Figure 10 (26 months).

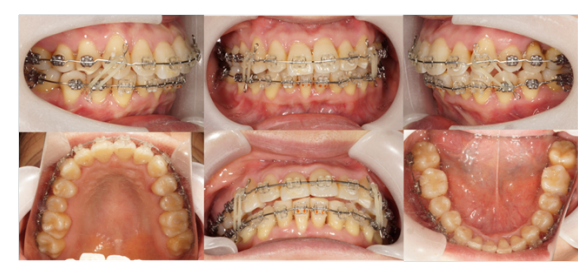

Figure 11.
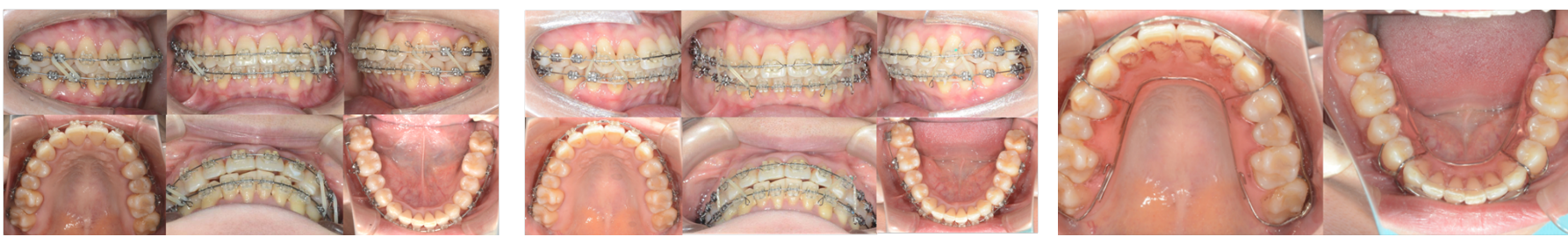

Figure 12.

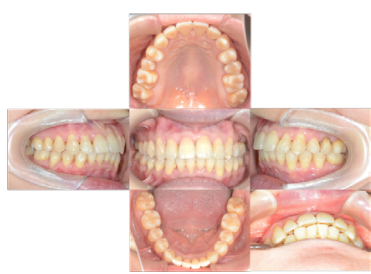

Table 2.

\begin{tabular}{|l|l|l|l|l|l|}
\hline & After & Before & Mean. & S.D. & Diff. \\
\hline Facial angle & 84.8 & 84.6 & 84.8 & 3.1 & 0.2 \\
\hline Convexity & 6.2 & 7.6 & 7.6 & 5 & -1.4 \\
\hline A-B plane & -5.7 & -6.8 & -4.8 & 3.5 & 1.1 \\
\hline Mandibular plane & 24.9 & 25.5 & 28.8 & 5.2 & -0.6 \\
\hline Y-axis & 62.7 & 63.0 & 65.4 & 5.6 & -0.3 \\
\hline Occlusal plane & 8.5 & 11.5 & 11.4 & 3.6 & -3.0 \\
\hline Interincisal & 103.2 & 124.0 & 124.1 & 7.6 & -20.8 \\
\hline L-1 to Occlusal & 38.2 & 26.8 & 23.8 & 5.3 & 11.4 \\
\hline L-1 to Mandibular & 111.8 & 102.8 & 96.3 & 5.8 & 9.0 \\
\hline U-1 to A-P plane & 12.5 & 9.2 & 8.9 & 1.9 & 3.3 \\
\hline FMIA & 43.3 & 51.7 & 54.6 & 6.5 & -8.4 \\
\hline FH to SN plane & 9.2 & 9.2 & 6.2 & 2.9 & 0.0 \\
\hline SNA & 78.5 & 79.0 & 82.3 & 3.5 & -0.5 \\
\hline SNB & 75.4 & 75.1 & 78.9 & 3.5 & 0.3 \\
\hline SNA-SNB diff. & 3.2 & 3.9 & 3.4 & 1.8 & -0.7 \\
\hline U-1 to N-P plane & 14.3 & 11.3 & 11.7 & 2.7 & 3.0 \\
\hline U-1 to FH plane & 120 & 107.7 & 111.1 & 5.5 & 12.3 \\
\hline U-1 to SN plane & 110.8 & 98.6 & 104.5 & 5.6 & 12.2 \\
\hline Gonial angle & 120.2 & 120.1 & 122.2 & 4.6 & 0.1 \\
\hline Ramus inclination & 84.7 & 85.3 & 87.1 & 4.4 & -0.6 \\
\hline
\end{tabular}

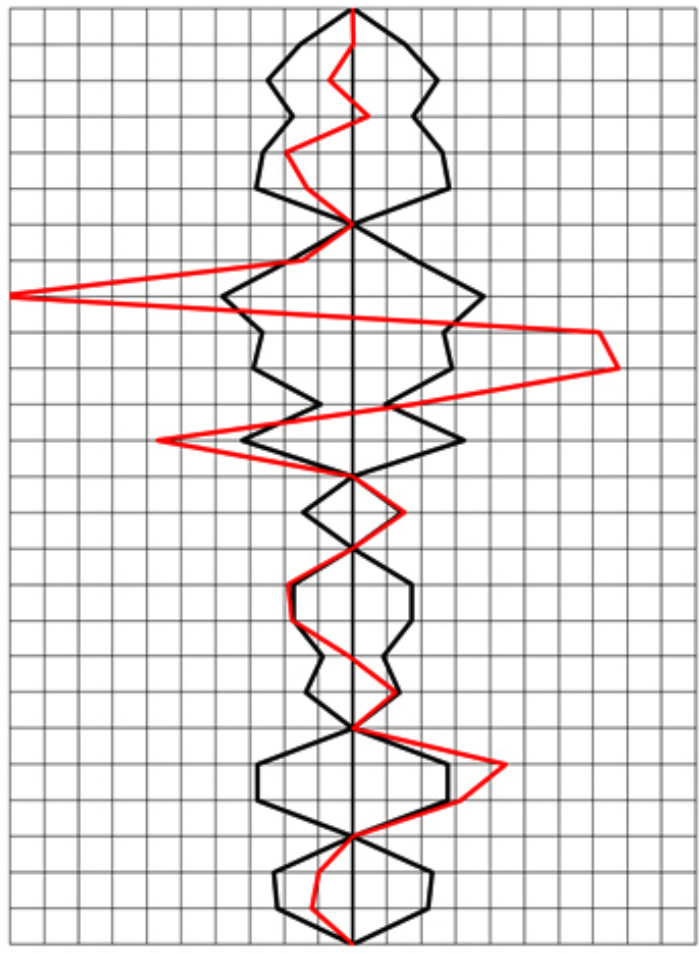

\#33, and \#34 to obtain better interdigitation (Figure 10). Finally, the fixed orthodontic appliances were removed, and upper circumferential and lower Hawley retainers were delivered (Figure 11, Figure 12).

As the dental problems of this case were resulted from space discrepancy, a non-extraction strategy was applied to maintain initial facial profile, Thus, the use of MEAW technique enabled to create more space for alignment. She still had a little protruding lips after treatment, but within acceptable limit. For this case, MEAW technique was used as the major approach to gain space, combined with stripping for more space and IME for anchor- age control and bite raising. Her deep bite was also corrected. Finally, a better dental occlusion and a favorable facial profile were achieved (Figure 13).

\section{Discussion}

For the treatment of this case, space creation was the most critical step. In addition to MEAW, interproximal stripping (the process of enamel reduction) was also used to gain sufficient space [3]. By gradual and gentle stripping, $0.25 \mathrm{~mm}$ each time (just meeting the mean width of PDL and the thickness of trimming strips), space was created for tooth alignment, thereby adjusting the tooth-size- 
Figure 13.

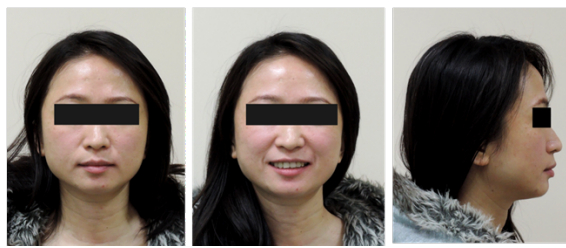

Figure 16.

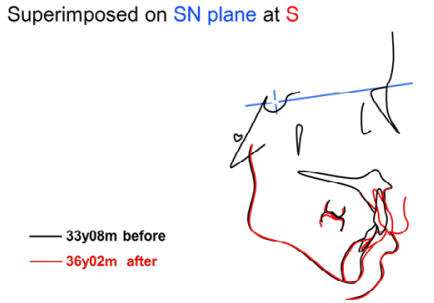

Figure 14.

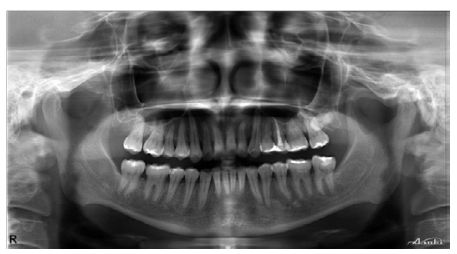

Figure 17.

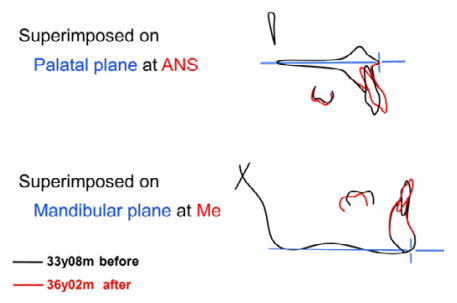

Figure 19.
Figure 15.
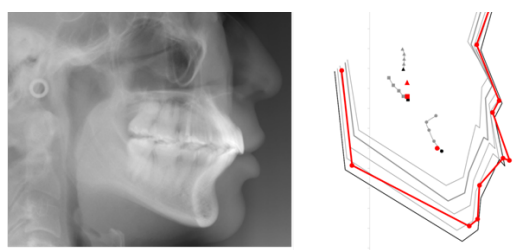

Figure 18.

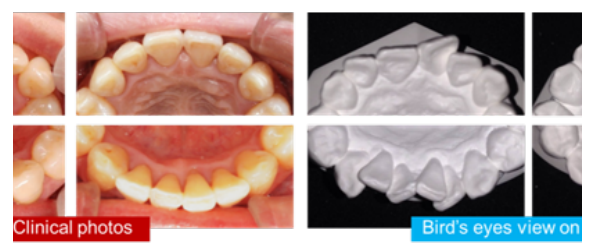

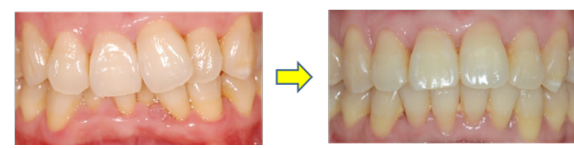

Table 3.

\begin{tabular}{|c|c|c|c|c|c|c|}
\hline$(\mathrm{mm})$ & 13 & 12 & 11 & 21 & 22 & 23 \\
\hline Initial & 8.5 & 7.3 & 8.2 & 8.5 & 7.2 & 8 \\
\hline Final & 7.7 & 7 & 8 & 8.2 & 7 & 7.6 \\
\hline Reduction & 0.8 & 0.3 & 0.2 & 0.3 & 0.2 & 0.4 \\
\hline$(\mathrm{mm})$ & 43 & 42 & 41 & 31 & 32 & 33 \\
\hline Initial & 6.7 & 5.8 & 5.5 & 5.5 & 5.8 & 6.8 \\
\hline Final & 6.5 & 5.5 & 5 & 5 & 5.5 & 6.2 \\
\hline Reduction & 0.2 & 0.3 & 0.5 & 0.5 & 0.3 & 0.4 \\
\hline
\end{tabular}

Total amount of reduction

Upper : $2.2 \mathrm{~mm}$

Lower : $2.2 \mathrm{~mm}$

ratio for more harmonious display in a friendly way, not only to the patient but also the tooth pulp tissue (Figure 18, Figure 19, Table 3) [4].

MEAW was used as the major approach for space creation. It can enable teeth upright and distal tipping, thereby providing the space for alignment [5]. However, the effectiveness of MEAW is highly dependent on the degree of a patient compliance, i.e. the use of intermaxillary elastics. Although an overall correction for all involved teeth was not achieved by using the MEAW technique in this case, it didn't result in unfavorable change in profile (Figure 20, Figure 21, Figure 22, Table 4).

One of the keys to maintain her initial pleasing profile and facial esthetics was sustain the lips position on lateral facial profile, in addition to position both the upper and lower lips related to the upper incisors [6]. This case had a discrepancy up to $8.0 \mathrm{~mm}$ in the upper arch. Even with MEAW technique, flaring out of the anterior teeth was inevitably presented. Stripping was therefore performed as mentioned above for creating more space to correct the inclination of anterior teeth during the stage of finishing and detailing. Eventually, a favorable result was achieved (Figure 23, Figure 24).

IME also played a indispensible role in the treatment process. By using Class II IME for bite raising, lower molars were extruded and led to occlusal plane "opening (clockwise rotation)", resulting in the leveling of lower anterior teeth was facilitated due to reduced interference from the uppers (Figure 25). On the other hand, with the use of short Class II IME, the effectiveness of MEAW for anchorage was reinforced. With serial step-up and tipback bends, the archwire resembles the appearance of curve, but possesses the opposite effects in mechanism. In an attempt to make MEAW function as we expected, the use of short Class II IME is feasible to prevent undesirable tooth movement (Figure 26). In finishing and detailing stage, "box" and "triangle" IME 
Figure 20.

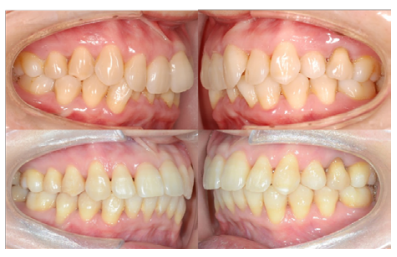

Figure 21.

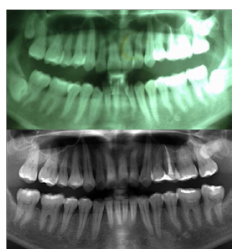

Table 4.
Figure 22.

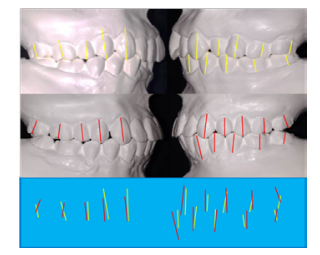

\begin{tabular}{|c|c|c|c|c|c|c|c|c|c|}
\hline \multicolumn{10}{|c|}{ Angulation change (Mesial $+^{\circ}$ \& Distal $-^{\circ}$ ) } \\
\hline 17 & 16 & 15 & 14 & 13 & 23 & 24 & 25 & 26 & 27 \\
\hline-32 & $-26^{\circ}$ & $9^{\circ}$ & $-8^{\circ}$ & $+7^{\circ}$ & $+6^{\circ}$ & $-7^{\circ}$ & $-12^{\circ}$ & $-15^{\circ}$ & $-28^{\circ}$ \\
\hline & & & & & 33 & 34 & 35 & 36 & 37 \\
\hline & & & & & $+11^{\circ}$ & $+10^{\circ}$ & $-3^{\circ}$ & $-12^{\circ}$ & $-21^{\circ}$ \\
\hline
\end{tabular}

Figure 23.

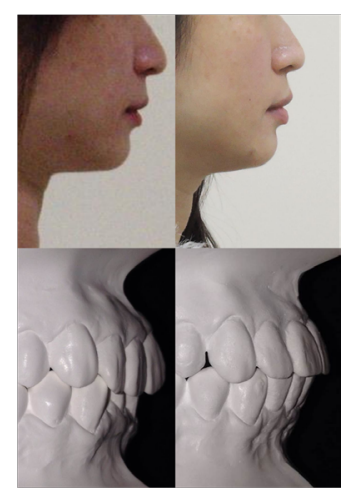

Figure 24.

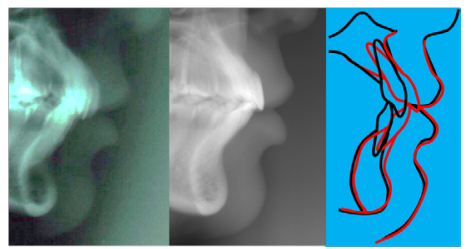

$\mathrm{U} 1$ to $\mathrm{FH}$ plane: $107.7 \circ \rightarrow 120.0^{\circ}$

U1 to A-Pog: $9.2 \mathrm{~mm} \rightarrow 12.5 \mathrm{~mm}$

L1 to Md Plane: $102.8^{\circ} \rightarrow 111.8^{\circ}$

Figure 26.

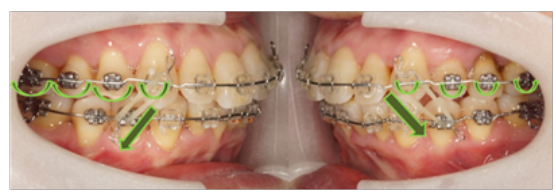

Figure 25.

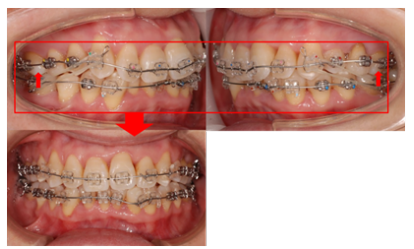

Figure 27.

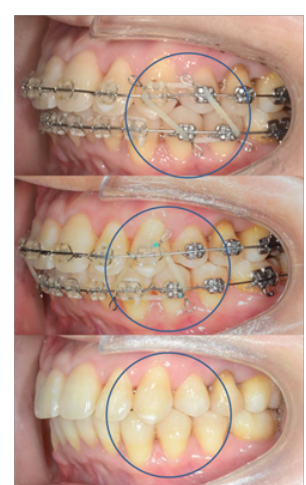

provided helpful support for interdigitation, combined with the high flexibility of ISW enabled this effectiveness more efficient (Figure 27).

\section{Acknowledgement}

"This work was supported by China Medical University and Hospital, Taichung City, Taiwan (Grant number: CMUH103REC3-048)."

\section{References}

[1]. Nance HN (1949) The removal of second premolars in orthodontic treatment. Am J Orthod 35(9): 685-696.

[2]. Sato $S$ (1994) Case Report: Developmental characterization of skeletal Class III malocclusion. Angle Orthod 64(2): 105-111.

[3]. Stroud JL, English J, Buschang PH (1998) Enamel thickness of the posterior dentition: Its implications for nonextraction treatment. The Angle Orthodontist 68(2): 141-146.

[4]. Bolton WA (1962) The clinical application of a tooth-size analysis. Am J Orthod 48(7): 504-529.

[5]. Karl-Friedrich Krey, Karl-Heinz Dannhauer (2012) Class II division 2 adult orthodontic treatment: A case report. J Stomat Occ Med 5(4): 177-182.

[6]. Talass MF, Talass L, Baker RC (1987) Soft-tissue profile changes resulting from retraction of maxillary incisors. Am J Orthod Dentofacial Orthop 91(5): 385-394. 